\title{
Relaciones filogenéticas de las serpientes del género Conopsis con base en la morfología
}

\author{
Phylogenetic relationships of the snake genus Conopsis based on morphology
}

\author{
Irene Goyenechea
}

Centro de Investigaciones Biológicas, Universidad Autónoma del Estado de Hidalgo. Apartado postal 1-69 Plaza Juárez, 42001 Pachuca, Hidalgo. México.

Correspondencia:ireneg@uaeh.edu.mx

\begin{abstract}
Resumen. El género Conopsis es endémico de México y se distribuye desde Chihuahua hasta Oaxaca. Recientemente se estudió su taxonomía, al modificarse su nomenclatura en los últimos años. Estas serpientes colúbridas de hábitos enterradores pueden reconocerse por diversas características, entre las que destaca un surco en por lo menos 1 de los 3 últimos dientes maxilares. El género cuenta con 6 especies reconocidas: C. acuta, C. amphisticha, C. biserialis, $C$. lineata, C. megalodon y C. nasus. Estudios previos intentaron resolver la filogenia del género con base en caracteres morfológicos, pero debido al polimorfismo encontrado en ellos no hubo resolución. En este estudio se reevaluaron los caracteres morfológicos de Conopsis usando el método de frecuencias generalizadas para obtener una filogenia de las especies que componen el género. Los resultados obtenidos indican que se pueden recobrar las relaciones al interior del género usando el programa Fastmorphology, y que C. nasus y C. megalodon son grupos hermanos que junto con $C$. biserialis y C. amphisticha forman un clado. Conopsis acuta resultó el taxón más basal del género . Las relaciones de los grupos externos no se resuelven del todo pero los análisis indican que el grupo de los Sonorini no se sustenta.
\end{abstract}

Palabras clave: Colubridae, México, máxima parsimonia, morfología.

\begin{abstract}
The snake genus Conopsis is endemic to Mexico and is found from Chihuahua to Oaxaca. It has been subject of study on its taxonomy due to recent modifications on its nomenclature. Now, this burrowing snake genus is diagnosed by several characters, standing out a groove in at least 1 of the 3-rear maxillary teeth. It comprises 6 known species: Conopsis acuta, Conopsis amphisticha, Conopsis biserialis, Conopsis lineata, Conopsis megalodon and Conopsis nasus. Previous works using morphological characters tried to solve the phylogenetic relationships between species in the genus but failed due to polymorphisms found in almost all characters studied. In this work those morphological characters were reevaluated using the generalized frequencies method and the relationships were recovered using the program Fastmorpholgy. Results show that $C$. nasus and C. megalodon are sister taxa and group together with C. amphisticha and C. biserialis. C. acuta remains as a basal species. Relationships within the external groups were partially resolved, but the Sonorini are not supported.
\end{abstract}

Key words: Colubridae, Mexico, maximum parsimony, fastmorphology.

\section{Introducción}

El género Conopsis se describió en 1858(Günther, 1858) $\mathrm{y}$ desde entonces ha tenido problemas nomenclaturales porque ha sido sinonimizada con el género Toluca y se ha confundido con otros géneros, como Pseudificimia, Toluca, Ficimia, Contia, Chionactis, Oxyrhina, Achirhina, Exorhina, Epirhina y Ogmius (Taylor y Smith, 1942).

Uno de los trabajos más importantes para el género es la revisión taxonómica que realizaron Taylor y Smith (1942), donde intentan esclarecer la identidad de los

Recibido: 01 noviembre 2007; aceptado: 15 enero 2009 géneros Conopsis y Toluca, así como la de cada una de sus especies, concluyendo que ambos géneros eran válidos.

A pesar de la revisión de Taylor y Smith (1942) siguió existiendo ambigüedad al tratar de identificar ejemplares de ambos géneros. En años recientes se realizó una nueva revisión taxonómica de los géneros y se modificó su nomenclatura, quedando dentro del género Conopsis todas las especies que se incluían en el género Toluca (Goyenechea, 2000; Goyenechea y Flores-Villela 2002).

Por lo tanto Conopsis se define ahora como un género de serpientes colúbridas que se distribuye en diversos tipos de vegetación, pero principalmente en bosques de pino y encino entre los 1700 y 3200 m smn. El género está compuesto 
por organismos de talla pequeña, aproximadamente de 300 $\mathrm{mm}$ de longitud hocico cloaca y se reconoce por varias características de escamas y coloración, pero sobre todo por poseer un surco por lo menos en 1 de los 3 últimos dientes maxilares (Goyenechea y Flores-Villela, 2002).

En estudios previos se hipotetiza que 1 de los 3 géneros, Ficmia, Pseudoficimia o Gyalopion, podría ser el grupo hermano de Conopsis (Stickel, 1943; Duellman, 1961y Hardy, 1975; Fig. 1).

Para Stickel (1943) el género Conopsis forma parte de las pequeñas culebras de Norteamérica o Sonorini sensu Dowling (1975), que incluye los géneros: Sonora, Procinura, Chionactis, Chilomeniscus, Toluca, Conopsis, Pseudoficimia y posiblemente Stenorrhina y Ficimia debido a una serie de caracteres relacionados con el cráneo y la dentición.

Otros autores (p. ej., Bogert y Oliver, 1945; Smith y Laufe, 1945) trataron de reconocer el grupo hermano de Conopsis, pero no es sino con Duellman (1961) y Hardy (1975) que se considera que Ficimia, Pseudoficimia y Gyalopion estaban muy relacionadas con Conopsis y Toluca. De hecho, Hardy (1975) hipotetizó que
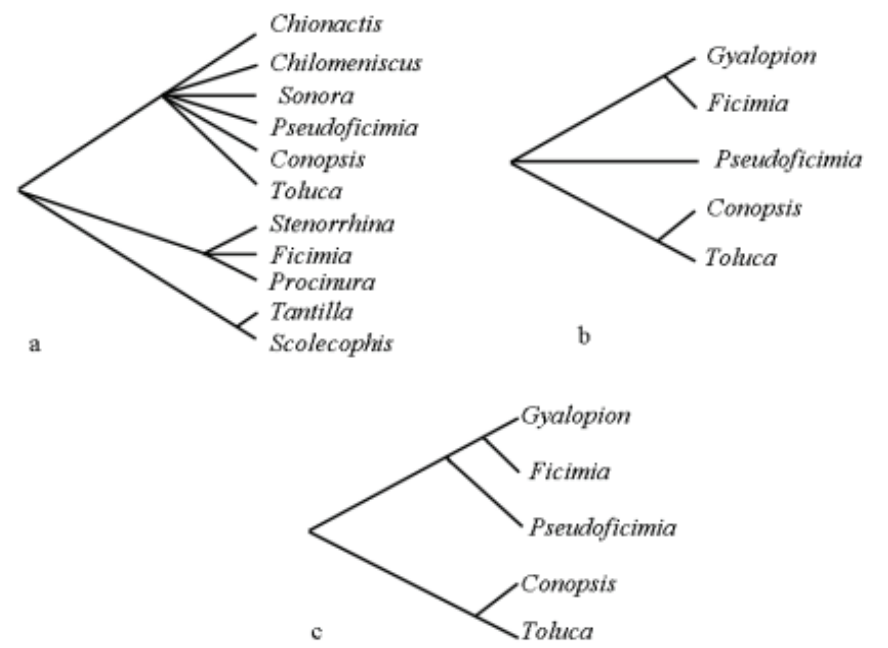

Figura 1. Algunas filogenias alternativas en forma de cladograma, para las especies del grupo de los Sonorini, dentro del que se sitúa Conopsis. a, filogenia propuesta por Stickel (1943); se observan 3 grupos sin resolución al interior de estos; el primero formado por Chionactis, Chilomeniscus, Sonora, Pseudoficimia, Conopsis y Toluca; el segundo, por Stenorrhina, Ficimia y Procinura, y el tercero, por Tantilla y Scolecophis; b, filogenia propuesta por Duellman (1961). Se propone que Ficimia y Gyalopion forman un grupo, Conopsis y Toluca forman otro y Pseudoficimia se sitúa en medio de ambos grupos; c, filogenia propuesta por Hardy (1975); se hipotetiza que Pseudoficimia es el grupo hermano del clado formado por Ficimia y Gyalopion, mientras que Conopsis y Toluca forman el grupo hermano de estos.
Pseudoficimia debería ser el grupo hermano de Ficimia y Gyalopion por poseer el peritoneo blanco a diferencia de Conopsis y Toluca que lo presentan negro (Hardy, 1975). No obstante, no se realizaron trabajos filogenéticos posteriores para conocer cuál de los 3 géneros podría constituir el grupo hermano de Conopsis.

Goyenechea (2000) realizó un estudio filogenético del género usando caracteres tanto morfológicos como moleculares de manera separada y 3 métodos diferentes (Kornet y Turner, 1999; Mabee y Humphries 1993 y Wiens, 1995) de codificación de los caracteres polimórficos para analizar la morfología de Conopsis. Sin embargo, debido al reducido número de caracteres morfológicos y a la cantidad de polimorfismos mostrados en cada uno de ellos, el resultado fue un cladograma politómico, por lo que se concluyó que la morfología por sí sola no puede resolver las relaciones entre las especies del género.

Smith y Gutberlet (2001) propusieron un nuevo método para analizar datos polimórficos con el programa Fastmorphology (Chang y Smith, 2001). Este método permite trabajar con caracteres polimórficos multiestado transformándolos de forma que se codifican para que puedan analizarse usando parsimonia mediante la técnica de codificación de frecuencias generalizadas (Smith y Gutberlet, 2001).

Este método ha probado ser útil en el análisis de caracteres morfológicos (Doan y Castoe, 2003), por lo que el objetivo de este trabajo es reevaluar los caracteres morfológicos de Conopsis analizados previamente para conocer las relaciones filogenéticas del género.

\section{Materiales y métodos}

Se analizaron los 16 caracteres morfológicos externos (Apéndice 1) propuestos por Goyenechea (2000) en un total de 1058 organismos (933 Conopsis y 125 Sonorini) para realizar un análisis cladístico de Conopsis y sus géneros relacionados. Los caracteres analizados fueron tanto binarios como multiestado cualitativos. Debido a que existe una gran cantidad de polimorfismos para cada carácter dentro de las especies estudiadas, se utilizó el método de codificación de frecuencias generalizadas (GFC) de Smith y Gutberlet (2001) para crear subcaracteres de frecuencias. Se utilizó el programa FastMorphology GFC (Chang y Smith, 2001) para transformar la matriz de datos crudos y obtener los subcaracteres de frecuencias, porque este programa utiliza la codificación generalizada de frecuencias para transformar los estados de carácter en un código accesible para hacer parsimonia.

Se utilizaron los datos de frecuencias estrictas para los caracteres no ordenados, los datos de frecuencias 
acumuladas para los caracteres ordenados, así como el esquema de pesaje desigual (USW) de acuerdo con lo sugerido por Smith y Gutberlet (2001). Con este método GFC, se emplean todos los datos polimórficos y no se descarta información a priori.

Se realizó un análisis filogenético usando PAUP* versión 4.0b10 (Swofford, 2002) con el criterio de máxima parsimonia, donde se incluyeron las 6 especies de Conopsis, de acuerdo con Goyenechea y Flores-Villela (2006), y un taxón representante de cada uno de los 8 géneros dentro de los Sonorini como grupo externo. Se realizó una búsqueda exacta con branch and bound y se calculó el soporte de los nodos con un remuestreo no paramétrico al azar con 1000 seudorréplicas (Felsestein, 1985) con 100 replicas de incorporación de taxa al azar, según los parámetros implementados por PAUP*.

\section{Resultados}

Los datos morfológicos proporcionaron 39 subcaracteres GFC de los cuales 15 fueron ordenados, 24 desordenados, 3 constantes, y 21 fueron parsimoniosamente informativos. La reconstrucción filogenética se obtuvo a partir de 12 árboles igualmente parsimoniosos de 463317 pasos con distintos pesos $(\mathrm{CI}=0.666, \mathrm{RI}=0.804 \mathrm{RC}=0.535$, $\mathrm{HI}=0.334$ ) cuyo consenso estricto se presenta en la figura 2. Se observan 2 grupos, uno que involucra a los grupos externos y otro que agrupa las especies de Conopsis, aunque la base del cladograma no se encuentra resuelta. El clado de Conopsis resulta monofilético en el $100 \%$ de los casos, al computarse tanto el consenso estricto como el de mayoría. Sin embargo, el remuestreo (Fig. 3) soporta este clado en el $73 \%$ de los casos. Al interior de este grupo,
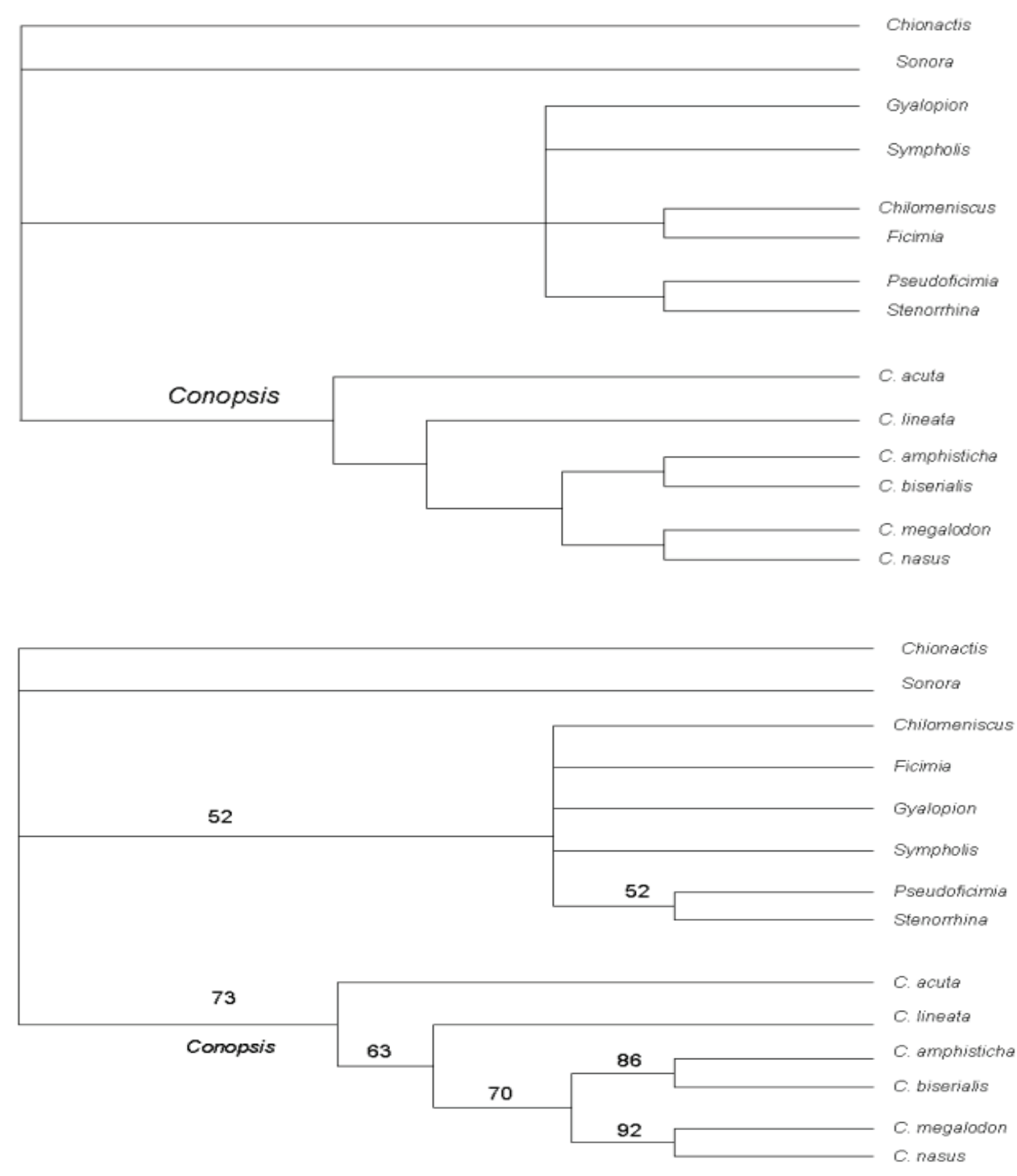

Figura 2. Cladograma de consenso estricto obtenido a partir de 12 árboles igualmente parsimoniosos de 463317 pasos con pesos distintos $(\mathrm{CI}=0.666$, $\mathrm{RI}=$ $0.804 \quad \mathrm{RC}=0.535, \quad \mathrm{HI}=$ $0.334)$.

Figura 3. Cladograma obtenido a partir del remuestreo de las ramas. El número por arriba de la rama indica el valor de soporte para el clado. 
C. nasus y C. megalodon (soportadas 92\%) son el grupo hermano de $C$. biserialis y $C$. amphisticha (soportadas $86 \%$ ). Este clado está soportado el $70 \%$, mientras que se observa que $C$. lineata es el grupo hermano del clado recién descrito en el 63\% y C. acuta es el taxón hermano de las restantes Conopsis.

Cabe señalar que a pesar de que el clado de los grupos externos se recobra en el consenso estricto, sólo se apoya con un valor de remuestreo del 52\%. Este grupo cuenta con muy poca resolución al interior del clado, pues sólo se observa a Pseudoficimia y Stenorrhina como grupos hermanos apoyados por 52\%. Fuera de este clado, se observan los géneros Sonora y Chionactis.

\section{Discusión}

Los cladogramas obtenidos tienen una resolución total en cuanto a las relaciones de las especies de Conopsis, con lo que se comprueba la eficacia del método propuesto por Smith y Gutberlet (2001) cuando se usan caracteres morfológicos y además polimórficos. Estos resultados muestran la estrecha relación de C. nasus y C. megalodon, los cuales comparten caracteres como las escamas prefrontales e internasales fusionadas, así como un patrón de coloración semejante. Por su parte, C. amphisticha y $C$. biserialis son grupos hermanos, pues comparten su patrón de coloración. Previo a este estudio y de acuerdo con los resultados de Goyenechea (2000) se pensaba que estos 2 taxones se agrupaban junto con C. acuta y C. lineata. Sin embargo, los resultados obtenidos muestran que $C$. lineata es el grupo hermano de este clado y que C. acuta es el taxón mas basal con relación a las demás especies de Conopsis.

Es interesante observar los géneros que conformaron el grupo externo de Conopsis, los Sonorini, sensu Stickel 1943, pues no se sustentan de acuerdo con el análisis realizado, tanto así que Sonora, género por el cual se nombró Sonorini al grupo, se encuentra fuera del mismo, y sólo quedan dentro del clado los géneros Chilomeniscus, Ficimia, Gyalopion, Sympholis, Pseudoficimia y Stenorrhina. Asimismo, se observa que Ficimia, Pseudoficimia y Gyalopion no forman un grupo como tanto Duellman (1961) como Hardy (1975) lo propusieron; por el contrario, Pseudoficimia se agrupa con Stenorrhina.

Desafortunadamente, debido a la falta de resolución en la base del cladograma, no se puede confirmar cuál de estos géneros está más emparentado con Conopsis, por lo que sigue siendo necesaria la evaluación de caracteres de DNAmt, para poder resolver sus relaciones, así como corroborar las de las especies de Conopsis recobradas mediante este análisis morfológico.

\section{Agradecimientos}

Este trabajo se realizó con el apoyo financiero de las siguientes instituciones: Museo Americano de Historia Nacional, mediante beca "Theodore Roosevelt", Comisión Nacional para el Conocimiento de la Biodiversidad mediante el proyecto "Filogenia de Conopsis Günther, Serpentes: Colubridae", El consejo Nacional de Ciencia y Tecnología mediante el proyecto "Filogenia molecular de los géneros de serpientes enterradoras de México" y la Universidad Autónoma del Estado de Hidalgo mediante proyectos apoyados en el Programa Anual Universitario y Programa Institucional de Investigación.

\section{Literatura citada}

Bogert, C. y J. A. Oliver. 1945. A preliminary analysis of the herpetofauna of Sonora. Bulletin of the American Museum of Natural History 83:297-426.

Chang, V. y E. N. Smith. 2001. FastMorphologyGFC Version $1.0 \mathrm{http}: / / \mathrm{www} 3 . u t a . e d u / f a c u l t y / e n s m i t h$

Doan, T.M. y T.A. Castoe. 2003. Using morphological and molecular evidence to infer species boundaries within Proctoporus bolivianus Werner (Squamata: Gymnophtalmidae). Herpetologica 59:432-449.

Dowling, H.G. 1975. Yearbook of herpetology 1974. Herpetological Information Search System, American Museum of Natural History, New York,

Duellman, W. E. 1961. The amphibians and reptiles of Michoacán, Mexico. Publication of the Museum of Natural History, University of Kansas 15:1-148.

Felsestein, J. 1985. Confidence limits on phylogenies: an approach using the bootstrap. Evolution 39:783-791.

Goyenechea, 2000. Filogenia del género Conopsis Günther, 1858 (Serpentes: Colubridae) con un análisis cladista del grupo de pequeñas culebras de Norteamérica. Tesis doctorado Facultad de Ciencias, UNAM, México, D. F. 179 p.

Goyenechea, I. y O. Flores-Villela. 2002. The taxonomic status of the snake genera Conopsis and Toluca (Colubridae). Journal of Herpetology 36:92-95.

Goyenechea, I. y O. Flores-Villela. 2006. Taxonomic summary of Conopsis Günther, 1858 (Serpentes: Colubridae). Zootaxa 1271:1-27.

Günther, A. 1858. Catalogue of colubrine snakes in the collection of the British Museum. Alden \& Mowbray, Alden, Oxford.

Hardy, L. W. 1975. Comparative morphology and evolutionary relationships of the colubrid snake genera Pseudoficimia, Ficimia and Gyalopion. Journal of Herpetology 9:107-152.

Kornet, D. J. y H. Turner. 1999. Coding polimorphism for phylogeny reconstruction. Systematic Biology 48:365-379.

Mabee, P. M. y J. Humphries. 1993. Coding polimorphic data: examples from allozymes and ontogeny. Systematic Biology 42:166-181.

Smith, E. N. y R. L. Gutberlet. 2001. Generalized frequency coding: a method of preparing polymorphic multistate characters for 
phylogenetic analysis. Systematic biology. 50:156-169.

Smith, H. M. y L. E. Laufe. 1945. Notes on a herpetological collection from Oaxaca. Herpetologica 3:1-19.

Stickel, W.H. 1943. The Mexican snakes of the genera Sonora and Chionactis with notes on the status of other colubrid genera. Proceedings of the Biological Society of Washington 56:109-128.

Swofford, D.L. 2002. PAUP* Phylogenetic analysis using parsimony (*and other methods), version 4.0b10. Sinauer, Sunderland, Massachusetts.

Taylor, E. H. y H. M. Smith. 1942. The snake genera Conopsis and Toluca. Kansas University Science Bulletin 28:325-363.

Wiens, J. J. 1995. Polymorphic characters in phylogenetic systematics. Systematic Biology 44: 482-500.

Wilkinson, M. 1992. Ordered versus unordered characters. Cladistics 8:375-385.

Apéndice 1. Descripción de los caracteres.

Los estados de carácter que se pudieron ordenar por intermediación (Wilkinson, 1992) se consideraron ordenados (O); cuando no fue posible, se consideraron desordenados (D). Los caracteres binarios no requieren orden.

Carácter 1. Prefrontales: presentes (0); fusionadas con internasales (1).

Carácter 2. Internasales: grandes (0); pequeñas (1).

Carácter 3. Loreal: presente (0); ausente (1).

Carácter 4. Frontal: pentagonal (0); hexagonal (1).

Carácter 5. Escama frontal en contacto con: prefrontales (0); internasales (1).

Carácter 6. Supralabiales, número (O): 7 (0); 6(1); 5(2).

Carácter 7. Escamas supralabiales antes del ojo: 2.5 (0); 3 (1).

Carácter 8. Infralabiales, número: 7 (0); 6 (1).

Carácter 9. Rostral (D): chata (0); punta (1); proyección hacia arriba (2); chata hacia abajo (3).

Carácter 10. Nasal: entera (0); ausente, fusionada con alguna escama (1).

Carácter 11. Color dorsal (D): pardo oscuro (0); pardo (1); canela (2); beige (3); verde olivo (4); gris (5); amarillo (6); naranja (7).

Carácter 12. Manchas dorsales (D): sin patrón (0); banda (1); hilera vertebral hexagonal clara (2); hilera vertebral hexagonal, teselado (3); teselado (4); 2 hileras, teselado (5); hilera vertebral hexagonal, 2 paravertebrales (6); 3 a 5 líneas, la vertebral más conspicua (7).

Carácter 13. Color ventral: crema (0); amarillo (1).

Carácter 14. Manchas ventrales (D): inmaculado (0); banda (1); hilera de puntos pareados (2); hilera de puntos intercalados (3); hilera de puntos centrales (4); hilera de puntitos pareados (5).

Carácter 15. Color del peritoneo: claro (0); oscuro (1).

Carácter 16. Tipo de reproducción: ovíparas (0); vivíparas (1). 